\title{
Das German Law Journal: Open Access als Element eines wissenschaftlichen Programms
}

\author{
Matthias Goldmann"
}

\section{A. Einleitung}

Open Access ist für das German Law Journal stets nicht nur Selbstzweck, sondern integraler Bestandteil seiner wissenschaftlichen Programmatik gewesen. Die wissenschaftlichen Ziele, die sich mit der Gründung des German Law Journals verbanden und zugleich eng mit der Globalisierung unserer Disziplin verknüpft sind, waren vor zwanzig Jahren nur über eine Online-Zeitschrift im Open Access-Format zu erreichen - ein damals gewagtes, wenngleich erfolgreiches Unterfangen (B.). Innovation verpflichtet jedoch auf Wandlungsfähigkeit. Da sich sowohl die Strukturen der Wissenschaft als auch der Markt für wissenschaftliche Zeitschriften seitdem verändert haben, hat das German Law Journal zu Beginn des Jahres 2019 den präzedenzlosen Schritt vollzogen, sich unter das Dach eines international renommierten Fachverlags zu begeben, selbstverständlich unter Beibehaltung seiner Politik des Open Access und der Gebührenfreiheit (C.). Es handelt sich dabei um ein Experiment mit offenem Ausgang. Ein entscheidender Faktor für den Erfolg wird die Umstellung der Finanzierung wissenschaftlicher Periodika vom herkömmlichne subskriptionsbasierten Modell auf alternative Finanzierungsformen sein, insbesondere durch eine Umschichtung der Mittel öffentlicher Bibliotheken. Neben der Förderung von autorenfinanzierten Open Access-Modellen durch Übernahme der Gebühren sollten Bibliotheken Abonnementsysteme für Open Access-Publikationen entwickeln, die allen offen stehen und die Verzerrungen durch traditionelle leserfinanzierte Zeitschriften bzw. autorenfinanzierten Open Access vermeiden. Insofern plant die Open Access-Politik der Universitätsbibliotheken bisher ein Stück weit am Bedarf vorbei (D.).

\section{B. Der Beginn: Globalisierung der Rechtswissenschaft durch Open Access}

Das German Law Journal geht auf eine Initiative von Peer Zumbansen und Russell Miller zurück. Im Jahr 1999 arbeiteten sie gemeinsam als wissenschaftliche Mitarbeiter am Bundesverfassungsgericht. In dieser Zeit der stieg gerade in Rechtspraxis und -wissenschaft das Interesse an Gerichtsentscheidungen aus anderen Rechtsordnungen spürbar an. Mehrere Entwicklungen zeichnen dafür verantwortlich: Zum einen die fortschreitende Europäische Integration, insbesondere in Gestalt des gerade in Kraft getretenen Vertrags von Amsterdam mit seinen Kompetenzerweite-

* Jun.-Prof. Dr. Matthias Goldmann, LL.M. (NYU), Goethe-Universität Frankfurt und Max-Planck-Institut für ausländisches öffentliches Recht und Völkerrecht, goldmann@jur.uni-frankfurt.de. Der Autor ist Co-Editor in Chief des German Law Journal, www.germanlawjournal.com. Dank an die Herausgeber sowie an Emanuel Towfigh für wertvolle Hinweise. 
rungen, gerade im Bereich der inneren Sicherheit; ferner die Globalisierung, die in Form der Welthandelsorganisation, des brandneuen Internationalen Strafgerichtshofs und der exponentiell ansteigenden Zahl investitionsrechtlicher Schiedsverfahren eine dezidiert rechtliche Seite zeigte und einerseits als Fortschrittsgeschichte empfunden, andererseits spätestens seit den Protesten von Seattle im Jahr 1999 der Kritik weiter Bevölkerungskreise ausgesetzt war; schließlich, eng verknüpft mit den vorgenannten Faktoren, der Aufstieg von Demokratie, Rechtsstaat und insbesondere der Menschenrechte als Kernelemente einer Globalverfassung. ${ }^{1}$

Diesen Tendenzen zum Trotz stellten Peer Zumbansen und Russell Miller fest, dass die Entwicklung von Gesetzgebung und Rechtsprechung im juristischen Schrifttum immer noch weitgehend abgeschottet von transnationalen Einflüssen rezipiert und kommentiert wurde. Eine Beobachtung, die letztlich nicht weiter erstaunlich war. Denn es gab keine Möglichkeit, in kurzer Frist an wichtige Entscheidungen aus anderen Jurisdiktionen in einer allgemein zugänglichen Sprache zu gelangen.

Dazu kamen aber Vorbehalte, ob expliziter oder impliziter Art, gegen eine vergleichend vorgehende, international offene Rechtswissenschaft. Globalisierung war auch für die Rechtswissenschaft ein schmerzhafter Prozess. Dies galt besonders dort, wo sie mit dem auch nur teilweisen Verzicht auf die Muttersprache erkauft werden musste. Schließlich ist die Sprache das Handwerkszeug der Rechtswissenschaft. Nicht jede* $r$ mag die Bereitschaft aufbringen, dieses Handwerkszeug mitten im Berufsleben noch einmal maßgeblich zu erneuern. Doch sollte man hinter der Sprache die mit der Internationalisierung der Rechtswissenschaft verbundenen Machtfragen nicht übersehen. Welches Gericht setzt in dem sich entspinnenden „judicial dialogue“ den Standard? Wer schreibt das maßgebliche Lehrbuch? Wessen Entscheidungen oder Schriften verlieren schneller an Wert als gehofft? Und vor allem: Ist es möglich, einen universellen Konsens über bestimmte Fragen zu erreichen, ohne dass gewisse Stimmen eine hegemoniale Stellung beanspruchen bzw. andere marginalisiert werden?

In dieser Situation gaben Peer Zumbansen und Russell Miller durch ihre Tat eine klare Antwort. Sie initiierten einen zu Beginn zweiwöchentlich erscheinenden Newsletter mit Besprechungen vornehmlich deutscher Gerichtsentscheidungen und Rechtsentwicklungen. Das Abkapseln gegenüber transnationalen Einflüssen erschien als aussichtsloses Unterfangen. Der Dominanz von Englisch als sich abzeichnender Wissenschaftssprache sollte aber nicht auch die Dominanz der Inhalte

1 Vgl. Mattias Kumm, "Kosmopolitischer Staat und konstitutionelle Autorität: Eine integrative Konzeption Öffentlichen Rechts", in Andreas Voßkuhle, Christian Bumke und Florian Meinel (Hrsg.), Verabschiedung und Wiederentdeckung des Staates im Spannungsfeld der Disziplinen (Duncker\&Humblot, Berlin 2013) 245-266. Zum europäischen Ursprung der Konstitutionalisierungsdebatte: Matthias Goldmann, „Hopes of Progress: European Integration in the History of International Law“, MPIL Research Paper No. 2018-26 (2018), https://dx.doi.org/10.2139/ssrn.3262453 (abgerufen am 25.2.2019). 
aus dem anglophonen Sprachraum folgen. Das Ergebnis war eine offene, emphatische Internationalität, die den eigenen Standpunkt und die Existenz von Differenzen jedoch nicht leugnet. Sie ist seit Beginn untrennbar mit dem German Law Journal verbunden.

Beim Newsletter blieb es nicht lang. Innerhalb weniger Nummern entpuppte sich das German Law Journal zur elektronischen Fachzeitschrift. Das Open Access-Format folgte dabei den sowohl den Notwendigkeiten als auch der Überzeugung. Zum einen bot sich kein traditionelles Zeitschriftenformat an. Die meisten Zeitschriften hatten ihren Schwerpunkt auf dem nationalen Recht. Rechtsvergleichende Publikationen wurden nur von denen sich speziell als rechtsvergleichend verstehenden Wissenschaftler"innen rezipiert. Dazu kamen Probleme bei der Verbreitung. Nur wenige amerikanische Fakultätsbibliotheken besorgen ausländische Fachpublikationen in nennenswertem Umfang. Nicht nur der Preis, sondern auch mangelnde Sprachkenntnisse standen größerer Verbreitung entgegen. Lange Produktionszyklen traditioneller Papierzeitschriften und hohe Kosten machten die Neugründung einer solchen Zeitschrift vollends unattraktiv. Online-Portale waren entweder noch gar nicht vorhanden, oder wie die Zeitschriften streng nach Sprache bzw. Rechtsordnung strukturiert und außerhalb des betreffenden Raums oft nicht zugänglich. Mithin ließ sich das wissenschaftliche Ziel des German Law Journals eigentlich nur durch eine frei zugängliche Online-Plattform erreichen. Nicht die nur schwer änderbaren Beschaffungsentscheidungen, nicht die Strukturen des kommerziellen juristischen Publikationsmarkts, sondern das Interesse an gleichberechtigtem transnationalem Austausch über Rechtsthemen sollte das German Law Journal leiten.

Der eingeschlagene Weg erwies sich als höchst erfolgreich. Innerhalb weniger Jahre etablierte sich das German Law Journal zu einer führenden Fachzeitschrift. Das zehnjährige Bestehen im Jahr 2009 feierte man bereits mit einem Festakt in Anwesenheit der Bundesministerin der Justiz. ${ }^{2}$ Der Wissenschaftsrat hob in seinem vielbeachteten Bericht zur Lage der Rechtswissenschaft aus dem Jahr 2012 den Beitrag des German Law Journals zur Steigerung der Sichtbarkeit der deutschen Rechtswissenschaft besonders hervor. ${ }^{3}$ Im Jahr 2018 rangierte das German Law Journal bei Google Scholar gemessen an den Zitaten jeweils auf Platz 3 weltweit in den Kategorien „International Law“ und „European Law“. ${ }^{4}$ Die auf einer Tagung zu Open Access in Frankfurt a. M. im Herbst 2018 vonseiten gut informierter Kreise

2 Dokumentiert in Russell A. Miller, Peer Zumbansen (Hrsg.), Comparative Law as Transnational Law: A Decade of the German Law Journal (Oxford University Press 2012).

3 Wissenschaftsrat, Perspektiven der Rechtswissenschaft in Deutschland. Situation, Analysen, Empfehlungen (2012), S. 70 (abrufbar unter https://www.wissenschaftsrat.de/download/archiv/2558-12.pdf).

4 Nachweise https://scholar.google.de/citations?view_op=top_venues\&hl=de\&vq=soc_internationallaw bzw. https://scholar.google.de/citations?view_op=top_venues\&hl=de\&vq=soc_europeanlaw (abgerufen am 25.2.2019). 
einhellig kolportierte Beobachtung, Open Access-Publikationen würden in Berufungsverfahren als „Malus“ gewertet, bietet insofern zwar Anlass zur Sorge - aber doch wohl eher um die Zukunft der deutschen Rechtswissenschaft denn um diejenige des German Law Journals. Es sei jedoch hinzugefügt, dass die Herausgeber des German Law Journals keine negativen Erfahrungen solcher Art bekannt sind. Zwar mag es Autoren geben, die ihre Manuskripte lieber bei einer stärker spezialisierten oder höher gerankten Zeitschrift einreichen, aber in Zeiten schrumpfender Bibliotheksbudgets bei gleichzeitig steigender Zahl wissenschaftlicher Publikationen muss man den Autor*innen die Vorteile von Open Access für die Rezeption ihrer Texte nicht weiter erläutern.

Thematisch steht das German Law Journal Beiträgen aus sämtlichen juristischen Fachsäulen offen. Mit anfangs zwölf, mittlerweile sechs bis acht Ausgaben pro Jahr kann es ein breites Themenspektrum abdecken, wenngleich ein gewisser Schwerpunkt auf dem Europarecht, dem Völkerrecht und der Verfassungsvergleichung liegt. Methodisch gesehen liegt das Augenmerk auf vergleichenden, interdisziplinär informierten arbeiten. Ein transnationaler Rechtsdiskurs kann nur fruchtbar geführt werden, wenn er von simplen „legal transplants“ Abstand nimmt und das Recht umfassend in seinem sozialen Kontext studiert. ${ }^{5}$ Dies schließt provokante, kontraintuitive und experimentelle Ansätze ausdrücklich ein. Diese Publikationsphilosophie setzt das German Law Journal dadurch um, dass der von eingehenden Manuskripten zu durchlaufende Peer Review ausschließlich vom internationalen Kreis der Herausgeber vorgenommen wird, die sich dem Journal und seiner Ausrichtung besonders verpflichtet fühlen. Daraus entsteht keine Gesinnungskontrolle und erst recht kein Sektierertum. Der Herausgeberkreis ist pluralistisch zusammengesetzt und erneuert sich behutsam alle paar Jahre, um sich eine gute fachliche, geographische und altersmäßige Durchmischung sowie eine Vielfalt der Perspektiven zu bewahren. Der Frauenanteil unter den Herausgebern liegt derzeit bei ca. $45 \% ;^{6}$ der Anteil der Autorinnen lag im Zeitraum 2014 bis 2016 bei 38\%.

Organisatorisch beruht das German Law Journal auf früher zwei, jetzt einem Team von Student Editors, welches an der Law School der Washington and Lee University in Lexington, Virginia, angesiedelt ist. Die Studierenden setzen sich während zwei Jahren mit großem Einsatz für das Journal ein. Sie redigieren sämtliche Manuskripte sprachlich und stilistisch - unverzichtbar für ein Journal, dessen Autor*innen mehrheitlich nicht Englisch zur Muttersprache haben. Darüber hi-

5 Programmatisch insofern der Aufsatz des Gründungsherausgebers Peer Zumbansen, „Comparative Law's Coming of Age? Twenty Years after Critical Comparisons“, German Law Journal 6 (2005) 1073.

6 Vgl. dagegen die Durchschnittswerte für das Fach in Ute Sacksofsky und Carolin Stix, „Was lange währt und immer noch nicht gut ist: Zur Repräsentanz von Frauen in der Wissenschaft vom Recht", Kritische Justiz 51 (2018) 464-474. 
naus sorgen sie für die technische Aufbereitung der Manuskripte, insbesondere die Beachtung der strengen amerikanischen Standards für das Zitieren juristischer Autoritäten. ${ }^{7}$ Dies erfordert mitunter viel Feingefühl. Nicht jeder der befassten Studierenden hat immer ein gutes Verständnis dafür, wann ein Nachweis erforderlich oder ein Eingriff berechtigt ist, und nicht jede* $\mathrm{r}$ Autor*in bringt die Geduld auf, die der für europäische Autoren ungewöhnliche Zitierstil erfordert. Auch dass Studierende die Arbeitsergebnisse von Wissenschaftlern überprüfen, welche ihnen im Rang deutlich überlegen sind, erfordert mitunter Gewöhnung. Meistens glückt die Zusammenarbeit jedoch hervorragend, so dass die Publikation im German Law Journal für beide Seiten zu einer transnational bereichernden Erfahrung wird.

\section{Die Gegenwart: Veränderungen und Einzug unter ein Verlagsdach}

Das Internet als sichtbarster Teil der mit der Globalisierung einhergehenden kommunikativen Revolution verbilligt und beschleunigt die Verbreitung von Innovationen. Was heute noch in Stein gemeißelt erscheint, kann schon morgen überholt sein. Während „Goldtammer's Archiv für Strafrecht“ sich in über 160 Jahren seiner Existenz kaum verändert hat, gilt für die Teilnehmer am transnationalen Rechtsdiskurs das Gegenteil. Dieser ändert sich parallel zu seinem Kommunikationskanal, dem Internet. Mit dem Übergang zum Web 2.0 schlug die Geburtsstunde juristischer Blogs und damit einer neuen Form der nahezu tagesaktuellen Verbreitung und Kommentierung von Informationen über aktuelle Rechtsentwicklungen. Ein Online-Medium wie das German Law Journal, letztlich ein Kind des Web 1.0, geriet dadurch unter Innovationsdruck.

Das German Law Journal stellte sich dieser Herausforderung, indem es sich auf seine Stärken besann. Dies bedeutete erstens eine Orientierung an Grundlagenforschung, und zweitens eine Spezialisierung auf Sonderhefte (Special Issues). Zwar schließt die Grundlagenorientierung nicht aus, dass wichtige Entscheidungen einflussreicher Gerichte im German Law Journal besprochen werden. Allerdings verändern sich hierfür die Maßstäbe. Nicht die informatorische Grundversorgung, sondern die vertiefte Kritik, der Vergleich und die Einordnung in einen größeren Rahmen stehen nunmehr im Zentrum der Aufmerksamkeit. Insofern bedeutet der bewusste Verzicht auf ein begleitendes Blog, welches allein schon angesichts der thematischen Breite des German Law Journals uferlos zu werden gedroht hätte, die Besinnung auf den Mehrwert wissenschaftlicher Reflexion mit Distanz zum Tages-

7 Harvard Law Review Association, The Bluebook. A Uniform System of Citation, 20. Aufl. (1991); inzwischen als Online-Ausgabe verfügbar. 
geschehen. Dazu gehören auch ganze Special Issues zu einzelnen Entscheidungen aber eben unter der Maßgabe einer langfristig ergiebigen Dokumentation. ${ }^{8}$

Special Issues haben sich daneben als neuer Markenkern des German Law Journal etabliert. Dahinter stehen mehrere Entwicklungen. Zum einen haben viele Autoren eine starke Präferenz für Zeitschriftenartikel gegenüber Sammelbandbeiträgen. Sammelbandbeiträge setzen zunächst die Beschaffung des Bands durch eine Bibliothek voraus, ob in der elektronischen Ausgabe oder in Druckfassung. Eine Zeitschrift - erst recht eine im Open Access-Format - hat dagegen bereits ihre Abonnenten. Die Rezeption von Buchkapiteln behindert ferner die vergleichsweise schlechte Erschließung von Sammelwerken durch Suchmaschinen und Kataloge. Während z.B. Google Scholar ein nahezu vollständiges Verzeichnis aller englischsprachigen Zeitschriftenartikel bietet, erscheinen selbst englischsprachige Sammelbandbeiträge dort oft nicht oder nur mit großer Verzögerung in den Ergebnislisten. Daran haben auch E-Books und Onlineplattformen großer Verlage bisher nicht viel geändert. Zudem werden Zeitschriftenbeiträge in den von Institution zu Institution bzw. Land zu Land unterschiedlichen Evaluationssystemen oft höher bewertet als Sammelbandbeiträge. Und - last but not least - geht ein Special Issue in der Regel mit einer erheblich geringeren Produktionszeit einher als ein Buch, gerade wenn es sich um eine lediglich online erscheinende Plattform handelt.

Das German Law Journal gibt heute zwischen zwei und vier Special Issues pro Jahr heraus. Dazu erscheinen fast in jedem Heft kleinere Special Sections, die einen thematischen Schwerpunkt vertiefen. Der thematische Reigen der Special Issues ist breit. Höhepunkte bildeten die Ausgabe aus Anlass des Todes von Jacques Derrida, ${ }^{9}$ eine der Mission des German Law Journals besonders entsprechende Ausgabe zur Transnationalisierung der Rechtslehre, ${ }^{10}$ oder ein Heft zur Reform eines anderen deutschen Exportschlagers, dem GmbH-Gesetz. ${ }^{11}$ Aus jüngster Zeit ist etwa das Sonderheft zur Euro-Krise anzuführen, welches unter dem vielsagenden $\mathrm{Na}$ men „Regeneration Europe“ vor allem junge Autor*innen versammelte, ${ }^{12}$ die Ausgabe zur Ukraine-Krise, welche vermutlich den Anlass für einen Hackerangriff kurz nach Veröffentlichung lieferte, der die Website des Journals vorübergehend lahmlegte (und den Aufbau einer erheblich besser gesicherten Website begünstig-

8 Vgl. z.B. den Special Issue zur ersten Vorlage des BVerfG an den EuGH im OMT-Verfahren: German Law Journal 15 (2014), Ausgabe 2, http://www.germanlawjournal.com/volume-15-no-02 (abgerufen am 25.2.2019).

9 „A Dedication to Jacques Derrida“, German Law Journal 6 (2015), Ausgabe 1, http://www.german lawjournal.com/volume-06-no-01 (abgerufen am 25.2.2019).

10 „The Transnationalization of Legal Education, German Law Journal 10 (2009), Ausgabe 7, http://w ww.germanlawjournal.com/volume-10-no-07.

11 „Reform of Germany's Private Limited Company (GmbH)“, German Law Journal 9 (2008), Ausgabe 9, http://www.germanlawjournal.com/volume-09-no-09 (abgerufen am 25.2.2019).

12 „Regeneration Europe“, German Law Journal 14 (2013), Ausgabe 5, http://www.germanlawjourna 1.com/volume-14-no-05. 
te), ${ }^{13}$ oder ein Doppel-Sonderheft zu Populismus und Konstitutionalismus aus Anlass des zwanzigjährigen Jubiläums im Frühjahr 2019. ${ }^{14}$ Seit 2016 werden die Special Issues aufgrund regelmäßiger Ausschreibungen in einem kompetitiven Verfahren von den Herausgebern ausgewählt. Der Zuspruch ist enorm; die Erfolgsquote aber dennoch etwas höher als bei freistehend eingesendeten Manuskripten. ${ }^{15}$

Dieser Wandel ließ den Wunsch nach höherer Professionalität aufkommen. Der mittlerweile erreichte Umfang des Betriebs machte den Einsatz einer Software für die Verwaltung der eingesendeten Manuskripte erforderlich. Ferner haben die wissenschaftlichen Verlage die elektronische Präsentation ihrer Zeitschriften zwischenzeitlich erheblich verbessert. Gerade auf dem englischsprachigen Markt erhält mittlerweile jeder Artikel einen DOI (einen „Digital Object Identifier“), ${ }^{16}$ über die er dauerhaft im Netz auffindbar ist. Eine elektronische Schnittstelle erlaubt die schnelle, problemlose Übernahme eines Werks in eine Literatursoftware (z.B. Endnote, Citavi), welche gerade im Ausland zum Teil deutlich stärker verbreitet sind. Der Einbau einer ORCID-Kennung („Open Researcher and Contributor ID“) ${ }^{17}$ erlaubt die eindeutige Identifikation der Autoren und korrekte Zurechnung von $\mathrm{Zi}$ tierungen.

Dazu kommt eine Entwicklung, über die man gewiss geteilter Meinung sein kann: der Triumphzug der Metrik, insbesondere des Impact Factors. Nun müssen über die Kritik dieser Messwerte nicht mehr viele Worte verloren werden. ${ }^{18}$ Sie ist inzwischen Gegenstand einer regelrechten Subdisziplin geworden. Tatsache ist jedoch, dass viele Kolleg*innen in anderen Ländern dem Impact Factor auf Gedeih und Verderb ausgeliefert sind. Dies betrifft insbesondere die an Universitäten in Mittel- und Osteuropa tätigen Kolleg*innen, welche traditionell zum Kern der Leser*innen- und Autor*innenschaft des German Law Journals zählen. Ihre Wissenschaftsadministrationen bewerten sie stur nach der Zahl ihrer Veröffentlichungen in Zeitschriften, welche aufgrund ihres Impact Factors auf einem anerkannten Index wissenschaftlicher Fachpublikationen gelistet sind. ${ }^{19}$ Aus Ostasien werden ähnliche Zwänge berichtet. Das macht die Publikation in einer Zeitschrift wie dem

13 „The Crisis in Ukraine“, German Law Journal 16 (2015), Ausgabe 3, http://www.germanlawjourna 1.com/volume-16-no-03 (abgerufen am 25.2.2019).

14 „Populism and Constitutionalism“, German Law Journal 20 (2019), Ausgaben 2 und 3, https:// germanlawjournal.com/volume-20-no-02-no-03/ (abgerufen am 20.9.2019).

15 Bei freistehenden Manuskripten beträgt die Annahmequote im Mittel etwa 15\%, bei Vorschlägen für Special Issues ca. 30\% mit abnehmender Tendenz.

16 Vgl. http://www.doi.org (abgerufen am 20.9.2019).

17 Vgl. http://www.orcid.org (abgerufen am 20.9.2019).

18 Für viele: Jerome K. Vanclay, „Impact factor: outdated artefact or stepping-stone to journal certification?" Scientometrics 92 (2012) 211.

19 Bekannt ist etwa der - nicht öffentlich - zugängliche Journal Citation Report von Thomson Reuters. 
German Law Journal für die Betroffenen zur reinen Liebhaberei. Ein solches Listing ist allerdings an erhebliche technische Voraussetzungen geknüpft.

Nun gibt es bestimmt für jede dieser aufgezählten Entwicklungen eine Do-It-Yourself-Alternative, ob kostenlos oder nicht. Nichts davon macht den Übergang zu einem Verlag zur zwingenden Notwendigkeit. Jedoch sind alle diese Alternativen mit ganz erheblichem Aufwand verbunden - Aufwand, den auch ein an die vierzig Köpfe zählendes Team aktiver Herausgeber nur unter großen Schwierigkeiten stemmen kann. Viel sinnvoller erscheint dagegen, dass die Herausgeber sich auf ihre Kernaufgabe konzentrieren, das Journal inhaltlich zu steuern und zu entwickeln, und die technisch-administrative Weiterentwicklung in die erfahrenen Hände eines kompetenten Verlags legen.

Im Fall des German Law Journals begünstigte das gestiegene Interesse englischsprachiger Verlage an Open Access die Entscheidung zugunsten eines Verlags. In der englischsprachigen Verlagswelt konzentriert sich das Interesse der Praktiker sehr stark auf Online-Datenbanken wie LexisNexis oder Westlaw. Monographien spielen eine untergeordnete Rolle, Kommentare sind inexistent, und die Ausbildungsliteratur erreicht aufgrund stärker differenzierter Kursangebote auch nicht die Bedeutung, die sie für die führenden deutschen Verlage hat. Allenfalls Handbücher und Lexika spielen eine gewisse Rolle. Das hat zur Konsequenz, dass wissenschaftlich orientierte englischsprachige Fachverlage ihr wissenschaftliches Portfolio nicht durch Einnahmen aus praxisrelevanten oder ausbildungsrelevanten Publikationen querfinanzieren können. Sie sind damit in hohem Maße von den Finanzierungsbedingungen der Wissenschaft abhängig. Und die geht in vielen Ländern in Richtung Open Access, allen voran durch die Forderung des European Research Council nach frei zugänglicher Publikation sämtlicher aus der Förderung resultierender Forschungsergebnisse. ${ }^{20}$ Eine Rolle mag dabei die unverfrorene Abonnementspolitik mancher Wissenschaftsverlage gespielt haben, welche von den Renditeerwartungen der Kapitalmärkte in unerträgliche Höhen getrieben wurde. Open Access gibt dabei selbst unter einem Modell, nach dem die Autor*innen für die Publikation sogenannte „Article Processing Charges“ bezahlen, woraufhin der $\mathrm{Zu}$ gang zur Publikation für die Leser*innen kostenfrei gewährt wird, ein gewisses $\mathrm{Maß}$ an Kontrolle an Autorenschaft und Wissenschaftsadministration zurück entscheidet doch nun endlich auch der Preis über die Wahl des Publikationsorts. Die vergleichsweise geringe Bedeutung derartiger Verlage für die Rechtswissenschaft hinderte freilich nicht die Erstreckung der Open Access-Politik auf die Rechtswissenschaft.

20 Vgl. European Research Council, Multi-Beneficiary Model Grant Agreement (2017), http://ec.europ a.eu/research/participants/data/ref/h2020/mga/erc/h2020-mga-erc-poc-multi_en.pdf (abgerufen am 25.2.2019), Art. 29.2. 
Anstatt das Modell der Finanzierung durch die Autoren fortzuschreiben und dadurch langfristig die Abonnementpreise zu kannibalisieren, entstand bei Cambridge University Press, einem gemeinnützigen Verlag im Eigentum der Universität von Cambridge, das Interesse an der Publikation einer originären Open AccessZeitschrift und der Entwicklung eines neuen, diesbezüglichen Geschäftsmodells. Statt ein neues Produkt in einem gesättigten Markt aufzulegen, erschien die Übernahme einer eingeführten Zeitschrift als aussichtsreichere Alternative. Aus diesen Gründen vereinbarten Cambridge University Press und das German Law Journal, mittlerweile in Gestalt eines gemeinnützigen Vereins rechtsfähig geworden, eine Kooperation. ${ }^{21}$ Ab 2019 erscheint das German Law Journal nun im Programm von Cambridge University Press, zunächst für fünf Jahre, mit Option auf Verdauerung. An der Politik des Journals ändert sich nichts; es bleibt beim freien, kostenlosen Zugang für Autor*innen und Leser*innen (sog. „diamond open access“). Gleichzeitig profitiert das Journal von der herausragenden verlegerischen und technischen Expertise von Cambridge University Press, dem Verbreitungsgrad seiner Plattform und seinem Renommee.

\section{Die Zukunft: Zur Open Access-Politik der Universitätsbibliotheken}

Leser mit Branchenkenntnis werden an dieser Stelle die Frage nach der Nachhaltigkeit dieses Modells stellen. Auch das German Law Journal muss langfristig seine Kosten einspielen, die für die Software, den Satz, das Marketing und die Verwaltung entstehen. Zwar handelt es sich um einen insgesamt überschaubaren Kostenrahmen. Doch die Einnahmen müssen erst erwirtschaftet werden. Angestrebt wird dafür eine Mischfinanzierung aus einer Reihe unterschiedlicher Quellen.

Ob die Finanzierung gelingt, wird sich zeigen. Es würde die Aussichten jedenfalls erheblich begünstigen, wenn eine neue Form der öffentlichen Dauerfinanzierung zur Verfügung stünde, vergleichbar den von überwiegend öffentlichen Wissenschaftsbibliotheken gezahlten Subskriptionspreisen für klassische Fachzeitschriften. ${ }^{22}$ Zwar finanziert das Bundesministerium für Bildung und Forschung eine Anschubfinanzierung für Open Access. Eine Dauerfinanzierung stünde zur Aufgabe des BMBF aber in Widerspruch, die außerhalb der Finanzierung außeruniversitärer Institutionen und des BAföG im Wesentlichen auf Projektförderung begrenzt ist. ${ }^{23}$

21 Vgl. „For Immediate Release: German Law Journal and Cambridge University Press to Enter Partnership as of 2019“, Presseerklärung vom 24.7.2018, http://www.germanlawjournal.com/partnershi pwithcambridge (abgerufen am 25.2.2019).

22 Vorschläge bei Ralf Schimmer, Kai Karin Geschuhn und Andreas Vogler, „Disrupting the subscription journals' business model for the necessary large-scale transformation to open access“, Max Planck Digital Library Open Access Policy White Paper (2015), http://dx.doi.org/10.17617/1.3 (abgerufen am 20.9.2019).

23 Vgl. Sybille Hinze, „Forschungsförderung und ihre Finanzierung”, in: D. Simon u.a. (Hrsg.), Handbuch Wissenschaftspolitik (Springer, Wiesbaden 2016) S. 413-428. 
Die Dauerfinanzierung wissenschaftlicher Publikationswege fällt hingegen wie bisher auch in die Zuständigkeit der Universitätsbibliotheken. Diese haben sich in den letzten Jahren immer mehr auf Open Access eingestellt, eine begrüßenswerte Entwicklung. Jedoch erschient ihre Strategie aus unserer Perspektive zumindest unvollständig. Grob gesagt, machen die meisten Universitätsbibliotheken drei Angebote im Bereich Open Access: Die Übernahme der Kosten für die Freischaltung der Werke universitätsangehöriger Wissenschaftler gemäß dem Modell des autorenfinanzierten Open Access; die Bereitstellung von Repositorien für die frei verfügbare Veröffentlichung von Werken ihrer Wissenschaftler, welche insbesondere für Dissertationen genutzt werden; sowie die Bereitstellung von technischen Plattformen für Open Access-Zeitschriften. Letztere Option ist besonders löblich - jedoch leider unvollständig. Das Gros der Kosten entsteht durch die Software für das Manuskriptmanagement, durch den Drucksatz der angenommenen Manuskripte, durch das Marketing sowie, wenn man kein Team ebenso fleißiger wie freiwilliger Student Editors hinter sich hat, durch die redaktionelle Bearbeitung der Manuskripte. Nichts davon ist in den von mir eingesehenen Angeboten der Universitätsbibliotheken enthalten. Sie ersparen einem lediglich Hosting-Kosten, einen verschwindenden Anteil an den Gesamtkosten. Man müsste entweder dauerhafte Finanzquellen eröffnen, welche es derzeit in Deutschland nicht in allgemeiner Form gibt, oder sämtliche Arbeit selbst stemmen, was auch große Lehrstühle an ihre Grenzen bringen dürfte, oder technisch, redaktionell und optisch auf dem Stand einer belächelten Amateurzeitschrift verharren. Die Universitätsbibliotheken greifen mit ihren Hosting-Angeboten für Open Access-Zeitschriften daher deutlich zu kurz.

Dass Bibliotheken hingegen die angesprochenen Funktionen der Verlage übernehmen und z.B. redaktionelle Services anbieten, ist weder zu erwarten noch zu befürworten. Hierfür fehlt es ihnen an verlagsspezifischer Kompetenz. Es ist damit an der traditionellen Arbeitsteilung festzuhalten, wonach die Wissenschaft zur Bibliothek kommt und nicht umgekehrt die Bibliotheken der Wissenschaft die Formate vorgeben.

In diesem Sinne bietet sich die Einführung von Finanzierungsmodellen für gänzlich frei verfügbare Open Access-Zeitschriften an, idealerweise auf der Ebene der Bibliotheksverbünde oder sogar im Rahmen einer Vereinbarung in Anlehnung an die Praxis der VG Wort, welche für das ganze Land gilt. Gegenüber dem autorenfinanzierten Open Access weisen solche Zeitschriften einen entscheidenden Vorteil auf: Die Möglichkeit, öffentlich finanzierte wissenschaftliche Ergebnisse zu publizieren oder zu rezipieren hängt nicht vom Standort oder der Finanzkraft des Publizierenden bzw. Rezipienten ab. Für einen transnationalen rechtswissenschaftlichen Austausch ist dies die Idealvorstellung. Die Verknüpfung von Publikation oder Rezeption mit finanzieller Potenz verzerrt letztlich die Inhalte der Wissenschaft. Da kön- 
nen die Gutachter*innen noch so fair und ausgewogen entscheiden, den ökonomischen Zwängen können sie sich langfristig kaum entziehen. Autor*innen von finanziell schlechter gestellten Institutionen bessere Konditionen zu bieten, setzt fatale Anreize auf der Ebene der Verlage. Ein solches Subventionswesen wird langfristig nicht ohne Auswirkung auf die Inhalte bleiben, zumindest auf das, was publiziert wird. Das ist für eine Disziplin wie die Rechtswissenschaft mit einer langen Geschichte von Hegemonie („herrschende Meinung“) und Marginalisierung ein inakzeptabler Zustand. ${ }^{24}$ Die Finanzierung von Open Access ist damit im Idealfall von den Inhalten vollständig zu entkoppeln. Der Weg dazu ist noch weit.

24 Beispiele hierzu z.B. bei Philipp Dann und Felix Hanschmann, Postkoloniale Theorien, Recht und Rechtswissenschaft", Kritische Justiz 45 (2012) 127, 129f. Vgl. ferner den Special Issue "Law's Pluralities", German Law Journal 18 (2017), Ausgabe 2 (hrsg. von Greta Olsen und Franz Reimer), http://www.germanlawjournal.com/volume-18-no-02 (abgerufen am 25.2.2019). 